\title{
Sistema autônomo de monitoramento da qualidade de ar aplicado a indústrias gesseiras utilizando arduíno e sensores de gás e poeira
}

\author{
Plácido C. S. Segundo* Danrley A. Santos** \\ Mykhael M. Canjão* Melyssa M. B. S. Carvalho* \\ Maria E. Batigalhia* Weslley K. R. Figueredo*** \\ Bruno S. dos Santos* João O. B. Diniz ${ }^{* * * *}$ \\ Aristófanes C. Silva ${ }^{* * *}$ \\ * Instituto Federal de Educação, Ciência e Tecnologia do Maranhão \\ (IFMA), (e-mail: [placido.segundo, bruno.serejo, \\ joao.bandeira]@ifma.edu.br; [mykhael.canjao,eduarda.batigalhia, \\ melyssa.barros]@acad.ifma.edu.br, \\ ** Faculdade do Piaúi (FAPI), (e-mail: danrleypow@gmail.com) \\ *** Universidade Federal do Maranhão (UFMA), (e-mail: \\ ari@nca.ufma.br)
}

\begin{abstract}
The environmental conditions of work in the installations of gypsum companies during the process of gypsum extraction have been associated with the incidence of respiratory diseases in workers of the sector. The dust that is dispersed in the air during the production process is loaded with particles of calcium sulfate dihydrate, a compound that can cause serious health damage if inhaled or in contact with the skin. This work proposes the development of a prototype, capable of monitoring the pollution rates present in areas of gypsum extraction and gypsum production. The objective of this work was to develop a low-cost automated solution using Arduino and gas/dust sensors capable of measuring pollution rates. The results were able to measure the degree of pollution dissipated by 3 companies, showing that it is feasible to use the system not only to verify the pollution but also to alert the population about the risks arising from the industries.

Resumo: As condições ambientais de trabalho nas instalações de empresas de gesso durante o processo de extração de gesso têm sido associadas à incidência de doenças respiratórias em trabalhadores do setor. A poeira que é dispersada no ar durante o processo de produção é carregada com partículas de di-hidrato de sulfato de cálcio, um composto que pode causar sérios danos à saúde se inalado ou em contato com a pele. Este trabalho propõe o desenvolvimento de um protótipo, capaz de monitorar as taxas de poluição presentes em áreas de extração de gesso e produção de gesso. O objetivo deste trabalho foi desenvolver uma solução automatizada de baixo custo utilizando Arduino e sensores gás/pó capazes de medir índices de poluição. Os resultados foram capazes de medir o grau de poluição dissipado por 3 empresas, mostrando ser viável usar o sistema não apenas para verificar a poluição, mas também para alertar a população sobre os riscos advindos das indústrias.
\end{abstract}

Keywords: Gypsum Industries; Autonomous system; Arduino; Sensors; Quality monitoring.

Palavras-chaves: Industriais gesseiras; Sistema de autônomo; Arduíno; Sensores;

Monitoramento de qualidade.

\section{INTRODUÇÃO}

No cenário atual os dispositivos são projetados para serem utilizados nas mais diversas áreas, com o avanço de hardware e softwares, foi possível o desenvolvimento de uma gama de produtos capaz de lidar com inúmeros problemas. O desenvolvimento de várias ferramentas autônomas inteligentes busca soluções que estejam diretamente ligadas ao cotidiano das pessoas (saúde, lazer, entretenimento, etc.) assim como nas rotinas empresariais (segurança, gestão de recursos, controle de acesso, etc.). Um exemplo disso seria o monitoramento de ambientes (umidade, temperatura, poluição, etc.) por sistemas autônomos inteligentes guiados através de sensores.

A poeira é constituída de toda partícula solida de qualquer tamanho, natureza ou origem, formada por trituração ou outro tipo de ruptura mecânica de um material original sólido, suspensa ou capaz de se manter suspensa no ar. A poluição atmosférica caracteriza-se pela presença de materiais ou formas de energia no ar que impliquem risco, 
dano ou moléstia grave as pessoas e bens de qualquer natureza emissora. No caso da exposição a um poluente interessa suas características: toxicológicas (capacidade de transformação, persistência ambiental e vias de penetração no organismo); características individuais dos expostos a esse poluente; e aspectos socioambientais do local da exposição (magnitude, duração e frequência, etc.) (da Indústria Carbonífera, 2000).

Exposição à poluição ambiental é considerada quando o fator de risco se encontra imediatamente próximo às vias de ingresso do organismo do indivíduo, ou seja, da respiração, alimentação, pele, placenta, etc. O gesso é um dos mais antigos materiais de construção fabricados pelo homem (Karni and Karni, 1995). Sua obtenção é relativamente simples e consiste no aquecimento a uma temperatura não muito elevada (cerca de $160^{\circ} \mathrm{C}$ ) e, posterior redução a pó, de um mineral relativamente abundante na natureza: a gipsita (PERES et al., 2001). A composição química da gipsita em estado puro corresponde à formula de $\mathrm{SO} 4 \mathrm{Ca} .2 \mathrm{H} 2 \mathrm{O}$, distribuindo-se percentualmente nos compostos $\mathrm{CaO}(32,60 \%)$, SO3 $(46,50 \%)$ e $\mathrm{H} 2 \mathrm{O}$ (20,90\%) (Karni and Karni, 1995).

Entre as propriedades físicas, é um mineral higroscópico que tem grande afinidade pelo vapor de água, sendo capaz de retirá-lo da atmosfera ou eliminá-lo de uma mistura gasosa. O gesso ou fosfo-gesso (CaSO4.2H20- sulfato de cálcio hidratado) é um subproduto da indústria de ácido fosfórico, derivado da reação de ácido sulfúrico sob a rocha fosfatada, realizada com o fim de produzir ácido fosfórico (Karni and Karni, 1995). A extração de gipsita, cuja produção se concentra no nordeste brasileiro, é capaz de provocar a destruição da vegetação do cerrado e caatinga que enfrentará grande dificuldade em sua recomposição, além de lançar na atmosfera uma grande quantidade de pó, provocando uma grande poluição (Andrade, 1994).

A principal substância, o Sulfato de Cálcio Dihidratado (CaSO4.2H20), pode causar diversos riscos à saúde. O contato e a absorção pela pele, a inalação e ingestão desta substância, podem causar diversos efeitos adversos à saúde humana. O contato com os olhos e com a pele pode causar irritação, causando vermelhidão e sensação de calor na pele. A substância quando inalada e ingerida pode causar prejuízos ao sistema respiratório e ao sistema digestivo (Oakes et al., 1982). Essa substância também pode causar efeitos adversos ao meio ambiente, como a contaminação do solo e de cursos de águas tornandoos saturados em cálcio e enxofre. O gesso não é um combustível, mas um oxidante fraco, podendo provocar a degradação de matéria orgânica e liberação de gases que podem ser combustíveis (Zhang and Li, 2016).

A cidade de Grajaú no interior do Maranhão possui como uma de suas principais atividades econômicas a produção de gesso. Considerada a segunda maior produtora de gesso no Brasil, em Grajaú produz-se em média 583 mil toneladas de gesso ao ano destinados à agricultura, construção civil, fábricas de cimento e outros setores gerando aproximadamente 1,8 mil empregos diretos e 5 mil empregos indiretos, com um faturamento anual acima de $\mathrm{R} \$ 51$ milhão de reais (Rabelo, 2014).

Muitos trabalhos e métodos são desenvolvidos para monitoramento da qualidade de poluentes em vários tipos de indústrias. O trabalho proposto por Alvarado et al. (2015) apresentou um método para monitorar partículas de areia usando sensores ópticos em um protótipo aéreo, o que mostrasse bastante robusto, porém um alto custo. Por sua vez, Simić et al. (2016) propuseram um sistema de monitoramento de gás e água utilizando multi sensores em áreas industriais para, o trabalho mostra-se robusto utilizando uma gama de sensores para verificar temperatura do ar, unidade relativa do ar, e presença de compostos voláteis. O trabalho proposto por Saad et al. (2013), apresenta um sistema para monitorar os parâmetros ambientais da qualidade do ar, como poluentes gasosos, partículas, temperatura e umidade que podem afetar o conforto, a saúde e o ambiente de trabalho interno dos ocupantes, se não forem mantidos adequadamente, o trabalho se mostra robusto na tarefa de monitoramento do ar.

Assim, observa-se que não apenas a industria, mas pesquisadores também vêm em busca de métodos autônomos de monitoramento da qualidade do ar. O trabalho aqui proposto, engloba as vantagens propostas na literatura, utilizando sensores alinhados a um protótipo para o monitoramento de industrias de gesso. Vale lembrar que estudos sobre a poluição pelo gesso em áreas de baixa renda, como o caso de Grajaú, são escassas. Desta forma, o protótipo proposto neste trabalho, busca utilizar mecanismos opensource $^{1}$ e de baixo custo a fim de viabilizar a produção de um sistema autônomo de monitoramento que seja barato e fácil de ser reproduzido.

Apesar da grande produção de gesso na cidade de Grajaú, atualmente não há nenhum tipo de controle de qualidade dos dejetos (seja gás ou poeria) lançados diretamente pelas empresas. Assim, o objetivo deste trabalho é desenvolver um protótipo de um sistema de qualidade para medir índices de poluição utilizando Arduino e sensores de gás/pó.

O presente trabalho engloba uma série de contribuições, dentre elas pode-se destacar: (a) um sistema capaz de monitorar a taxa de poluição atmosférica presente em áreas de concentração de gesso; (b) desenvolvimento de uma solução automatizada de baixo custo; e (c) um sistema com um método eficaz, capaz não só de medir a taxa de poluentes, mas alarmar contra a poluição gerada pela emissão de gesso. Assim, mostra-se um protótipo robusto, para auxiliar empresas na emissão de poluentes, assim, alarmando não só a população como também empresários para provimento de melhores serviços.

Além desta seção o trabalho ainda possui mais 4 seções. A Seção 2, descreve os mecanismos de hardware e software utilizadas para criação do sistema. Na Seção 3, é apresentado como foi desenvolvido o protótipo, para tanto mostrase os sensores e circuito utilizado e o funcionamento do mesmo. Os resultados são apresentados e discutidos na Seção 4. Por fim, a Seção 5 conclui o trabalho avaliando o mesmo e discorrendo sobre alguns passos futuros.

\footnotetext{
1 software de computador com o seu código fonte disponibilizado e licenciado com uma licença de código aberto no qual o direito autoral fornece o direito de estudar, modificar e distribuir o software de graça para qualquer um e para qualquer finalidade
} 


\section{MECANISMOS DE HARDWARE E SOFTWARE}

Esta seção, descreve os mecanismos necessários para o desenvolvimento do protótipo. Serão descritos os hardwares e softwares utilizados para criação do sistema de monitoramento da qualidade do ar nas indústrias de gesso.

A solução proposta para desenvolvimento do protótipo é composta, basicamente, da combinação de três tecnologias principais, um módulo Arduíno UNO integrado a sensores de gás e poeira, uma placa Raspberry PI 3 Model B com o sistema operacional Raspbian instalado e a ferramenta de desenvolvimento Node-RED que é utilizada na implementação do sistema responsável pelo monitoramento.

Arduino é uma plataforma open-source composta por duas partes principais: hardware que é o componente físico representado por uma placa de circuitos eletrônicos capaz de ser integrada a dados analógicos e digitais através de um processo conhecido como transdução, uma forma de conversão de energia detectada em outra forma de energia (Arduino, 2015); e o software, a parte lógica que pode ser utilizada para a implementação dos programas a serem executados pelo hardware. A escolha da plataforma Arduíno, partiu da facilidade proporcionada ao usuário, no que se trata a controle total de hardware e software, além da ampla quantidade e variedade de componentes que podem ser integrados de maneira prática e descomplicada.

O Raspberry PI é um microcomputador de baixo custo que possui, portando dimensões não muito diferentes a de um cartão de crédito, configurações similares a um computador comum, como: memória RAM, processador, interfaces USB e HDMI, além das tecnologias Bluetooth e WI-FI para comunicação wireless (Upton and Halfacree, 2014). No escopo deste projeto o Raspberry PI foi utilizado como um midleware, com a simples função de integrar o coordinator(Arduíno) ao Node-RED, uma ferramenta utilizada para o desenvolvimento do sistema responsável por processar os dados do ambiente obtidos via sensores, em um dashboard contendo gauge charts para a representação gráfica das informações (Upton and Halfacree, 2014).

O Raspbian é um sistema operacional otimizado para projetos de softwares embarcados utilizando a placa Raspberry PI baseado no Debian, uma distribuição Linux, bastante conhecida no mercado. Sua plataforma é opensource, ou seja, seu código fonte pode ser utilizado por qualquer indivíduo independente do propósito. No escopo do protótipo o Raspbian tem como funções principais possibilitar o desenvolvimento e execução do sistema com Node-RED e estabelecer a Comunicação serial entre o Arduíno e o Raspberry PI (Harrington, 2015).

Estabelecidos e descritos os mecanismos necessários, o próximo passo é a descrição do desenvolvimento do protótipo.

\section{DESENVOLVIMENTO DO PROTÓTIPO}

O desenvolvimento do protótipo, além dos mecanismos já citados, também fez uso de alguns sensores. O projeto deste protótipo foi elaborado no Instituto Federal do Maranhão (IFMA), no Campus Grajaú, onde, como citado na Seção 1, é um dos maiores produtores de gesso do Brasil. Além da motivação encontrada, o desenvolvimento do protótipo, tanto para criação como para teste do mesmo se torna mais eficiente, visto que o ambiente o qual foi desenvolvido apresentava características fundamentais para validação.

A seguir serão apresentados os sensores utilizados, a elaboração do circuito e, por fim, o funcionamento do protótipo.

\subsection{Sensores de gás e poeira}

Como já falado na Seção 2, alguns mecanismos de hardware e software foram utilizados para desenvolvimento do protótipo, além destes, alguns sensores foram empregados, a seguir serão listados:

- Sensor MQ-135: Modulo capaz de detectar diversos tipos de gases, dentre eles: Amônia, Dióxido de Carbono, Benzeno, Oxido Nítrico, fumaça e álcool. Possui uma alimentação de $5 \mathrm{~V}$ e sáıdas digital e analógica (Simić et al., 2016).

- Sensor MQ-09: Módulo capaz de detectar gases como Metano, Propano e Monóxido de Carbono. Possui uma alimentação de $5 \mathrm{~V}$ e saídas analógica e digital (Falohun et al., 2016).

- Sensor MQ-02: Módulo capaz de detectar fumaça e gases combustíveis como: GLP, Metano, Propano, Butano, Hidrogênio, Álcool, Gás Natural e outros inflamáveis. Possui uma alimentação de $5 \mathrm{~V}$ e saídas digital e analógica (Dewi et al., 2018).

- Sensor DSM 501A: Módulo responsável por identificar partículas de tamanho compacto, em geral, é capaz de detectar poeiras finas em partículas tão pequenas quanto 1 (micrômetro) e partículas que flutuam em um espaço de até $30 \mathrm{~m}$. Possui uma alimentação de $5 \mathrm{~V}$ e saídas analógica e digital (Sulistiyo and Suryono, 2016).

Além dos mecanismos de hardware e software e os sensores citados, adicionalmente foi necessária a utilização de uma mini-protoboard e jumpers para conexão dos componentes citados. A Figura 3.1 mostra um exemplo de miniprotoboard e jumpers.

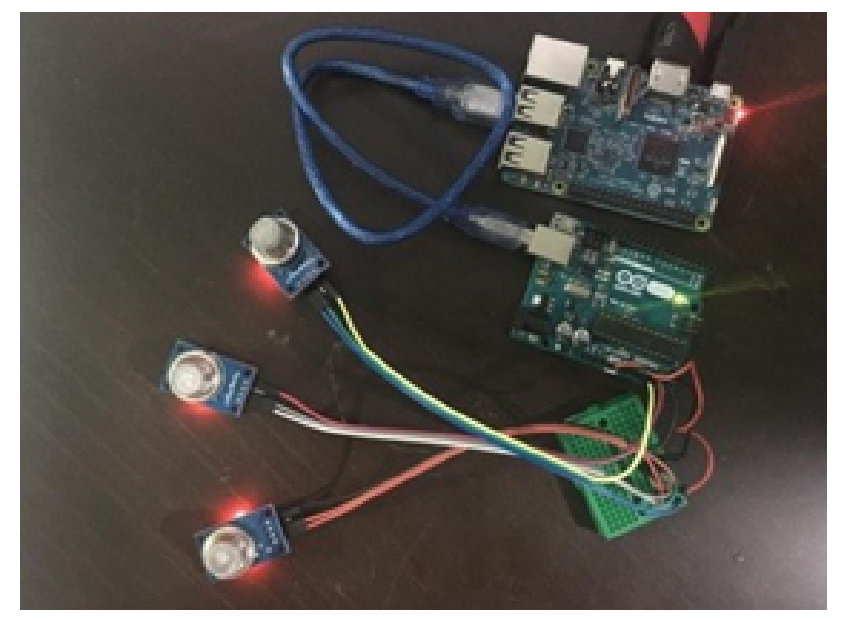

Figura 1. Exemplo de mini-protoboard e jumpers.

Definidos os sensores e protoboard, o próximo passo é a elaboração do circuito. A próxima subseção detalha como o circuito foi elaborado. 


\subsection{Elaboração do circuito}

O circuito do projeto foi esquematizado com auxílio de um software para prototipagem chamado Fritzing (Knörig et al., 2009). Como é possível observar na Figura 3.2, os componentes estão interligados à placa através dos pinos analógicos e alimentados por uma saída de energia de $5 \mathrm{~V}$ e uma saída GND. A placa Arduino por sua vez, está conectada ao Raspberry PI via conexão serial USB.

No esquema do projeto é possível verificar que o pino A0 está responsável por receber as leituras de dados realizadas pelo sensor MQ-135; o pino A1 está responsável por receber as leituras de dados realizadas pelo sensor MQ-2; o pino A2 está responsável por receber as leituras de dados realizadas pelo sensor MQ-9; e o pino A3, por sua vez, está responsável por receber as leituras de dados realizadas pelo sensor DSM 501A. A conexão dos sensores às suas respectivas portas pode ser observada conforme a Tabela 3.2.

\begin{tabular}{|c|c|}
\hline Sensores & Pinos \\
\hline MQ-135 & A0 \\
\hline MQ-2 & A1 \\
\hline MQ-9 & A2 \\
\hline DSM 501-A & A3 \\
\hline
\end{tabular}

Tabela 1. Relação entre sensores e pinos.

A próxima etapa após definir mecanismos, sensores e o esquema do projeto é entender o funcionamento em conjunto de todas as variáveis apresentadas. A seção que segue detalha o funcionamento do protótipo.

\subsection{Funcionamento do protótipo}

O sistema é baseado em leituras do ambiente realizadas pelos sensores integrados ao Arduino. Os dados coletados são enviados à placa Raspberry PI via conexão serial USB e em seguida são utilizados como parâmetro pela ferramenta Node-RED para a geração do dashboard contendo as informações processadas em forma de gráficos e gauge charts, como pode ser visto na Figura 3.

O dashboard pode ser visualizado através de uma tela touch screen ou projetada utilizando-se a interface HDMI do Raspberry PI. Para a integração do Arduíno ao NodeRED se fez necessário a identificação da porta exclusiva a ser utilizada na comunicação, através do comando "ls /dev/tty*" via terminal. Para o projeto foi utilizada a porta "/dev/ttyACM0". A configuração dos sensores em modo analógico permite que as medições sejam realizadas em unidades variando entre 0 e 1024 . Tais leituras podem ser convertidas para outras unidades de medida utilizando funções específicas.

No dashboard (Figura 3.3), são exibidos 4 gauge charts para representação das leituras dos sensores MQ-135, MQ09, MQ02 e DSM 501A respectivamente. Logo abaixo existe um outro gráfico medindo a variação de poeira por hora e um último gráfico medindo a variação de gases poluentes por hora.

Com os dados do dashboard medidos pelos 4 sensores, é possível avaliar de forma quantitativa os valores de poluentes de empresas gesseiras, e assim ter uma visão prática do grau de poluição que uma empresa está dispersando. Utilizando estes dados, as empresas podem agir de forma efetiva para uma tomada de decisão rápida de forma a minimizar a emissão de poluentes no ambiente.

\section{RESULTADOS E DISCUSSÃO}

O protótipo, assim que concluído, foi submetido a calibragem dos sensores e logo após a testes em campo. Foram selecionadas três empresas do polo gesseiro da cidade de Grajaú - MA para a realização dos testes. O protótipo foi instalado nas empresas durante o período de expediente das mesmas, em dias alternados de modo que cada empresa teve dados coletados de um dia completo de funcionamento conforme a Tabela 2. As empresas foram identificadas por Empresa X, Empresa Y e Empresa Z, respectivamente. A ordem das empresas foi definida pelo porte das mesmas iniciando pela menor. Os sensores operam em modo analógico que possuem variações entre $0-1023$, para tornar esses valores interpretativos, na Tabela 2 , foi utilizado valores em porcentagem.

Nos resultados dos testes pôde-se observar que o sensor MQ-02 responsável por detectar fumaça e gases combustíveis apresentou uma diferença mínima na média de valores coletados entre as empresas, apresentando um índice moderado de poluição.

O sensor MQ-09 apresentou dados semelhantes ao MQ02, com uma margem muito pequena de diferença entre os dados coletados nas empresas, também apresentando um índice moderado de poluição por Monóxido de Carbono, Metano e Propano.

Os dados coletados pelo sensor MQ-135 por sua vez apresentaram valores um pouco mais distinto, porém em uma margem não muito grande, apresentando um índice relativamente baixo de Amônia, Alcool, Dióxido de Carbono e Óxido Nítrico.

O sensor DSM 501A foi quem apresentou um maior índice de poluição mantendo uma relação de valores diretamente proporcional ao tamanho das empresas, quanto maior a empresa maior foi o índice de poluição detectado por este sensor que é responsável por monitorar a quantidade de poeira no ambiente. Os índices de poluição detectados pelo sensor DSM 501A foram bastante elevados.

Pôde-se constatar com os dados dos testes realizados que o sensor MQ-135 apresentou os menores índices de poluição; os sensores MQ-02 e MQ-09 apresentaram índices de poluição moderados; o sensor DSM 501A por sua vez apresentou índices altíssimos de poluição. Considerando que o principal composto responsável pela incidência de doenças respiratórias e de pele nas empresas dos polos gesseiros, o Sulfato de Cálcio Dihidratado (CaSO4.2H20), pode ser encontrado em grandes quantidades na poeira resultante do processo de extração da gipsita, assim, é alarmante os dados coletados nas empresas onde os testes foram executados. Visto que, os maiores índices de poluição identificados foram justamente os de poeira, monitorados pelo sensor DSM 501A. 


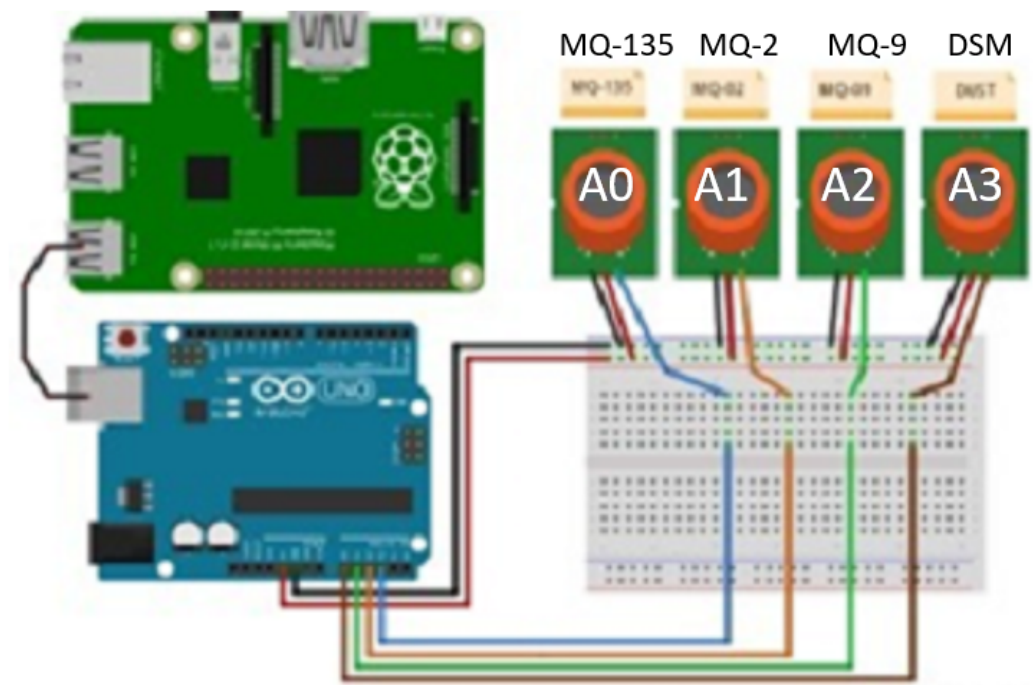

Figura 2. Esquema do protótipo.

\begin{tabular}{|l|l|l|l|l|}
\hline \multirow{2}{*}{ Empresa } & \multicolumn{4}{|c|}{ Media de valores coletados } \\
\cline { 2 - 5 } & MQ-02 & MQ-09 & MQ-135 & DSM 501A \\
\hline $\mathrm{X}$ & $53,95 \%$ & $60,99 \%$ & $23,80 \%$ & $82,89 \%$ \\
\hline $\mathrm{Y}$ & $53,76 \%$ & $61,77 \%$ & $26,58 \%$ & $85,23 \%$ \\
\hline $\mathrm{Z}$ & $53,95 \%$ & $62,33 \%$ & $27,95 \%$ & $89,34 \%$ \\
\hline
\end{tabular}

Tabela 2. Média de dados coletados por sensor para cada empresa.

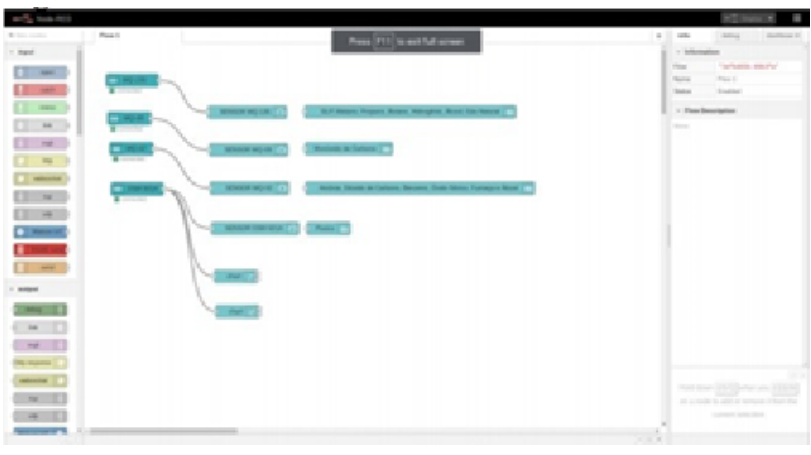

Figura 3. Desenvolvimento do sistema com Node-RED.

\section{CONCLUSÃO}

Neste trabalho foi desenvolvido um protótipo autônomo capaz de avaliar a qualidade do ar nas instalações do polo gesseiro na cidade de Grajaú-MA. O objetivo principal foi desenvolver uma solução de baixo custo capaz de monitorar a quantidade de poluentes gerados mediante a composição química na fabricação do gesso, através da utilização de sensores capazes de detectar a presença de poeira e gases poluentes como Metano, Dióxido de Carbono, Benzeno, Óxido Nítrico, Metano, Propano, Monóxido de Carbono, entre outros.

Pode-se afirmar que o protótipo desenvolvido funcionou dentro do esperado, uma vez que o sistema apresentou ser capaz de detectar as alterações ambientes no ar apresentando tais variações em um dashboard de informações. Através de testes realizados em campo, foi possível averiguar a eficácia do protótipo ao detectar a presença e apresentar as variações dos índices de poeira carregada de Sulfato de Cálcio Dihidratado (CaSO4.2H20) e outros gases também prejudiciais à saúde. Desta forma, o sistema se mostrou capaz de identificar locais com altos índices de poluentes e pode ser utilizado tanto com uma ferramenta auxiliar na prevenção de problemas de saúde, como um sistema para que as indústrias possam verificar seu grau de poluição e assim tomar decisões preventivas.

Como trabalhos futuros, propõe-se o uso de outros tipos de sensores, além de estender o trabalho para ser utilizado em outros tipos de industriais que despejem dejetos no ambiente.

\section{AGRADECIMENTOS}

Os autores agradecem ao Instituto Federal do Maranhão, em especial ao Campus Grajaú, onde foi elaborado o protótipo aqui apresentado.

\section{REFERÊNCIAS}

Alvarado, M., Gonzalez, F., Fletcher, A., and Doshi, A. (2015). Towards the development of a low cost airborne sensing system to monitor dust particles after blasting at open-pit mine sites. Sensors, 15(8), 19667-19687.

Andrade, M.C.d. (1994). O desafio ecológico: utopia e realidade. 504.06 AND.

Arduino, S.A. (2015). Arduino. Arduino LLC.

da Indústria Carbonífera, I.A. (2000). Ministério de minas e energia, departamento nacional de produção mineral. Brasília, ano $X V, 217 p$.

Dewi, S.S., Satria, D., Yusibani, E., and Sugiyanto, D. (2018). Design of web based fire warning system using ethernet wiznet w5500. In Proceedings of MICoMS 2017, 437-442. Emerald Publishing Limited. 


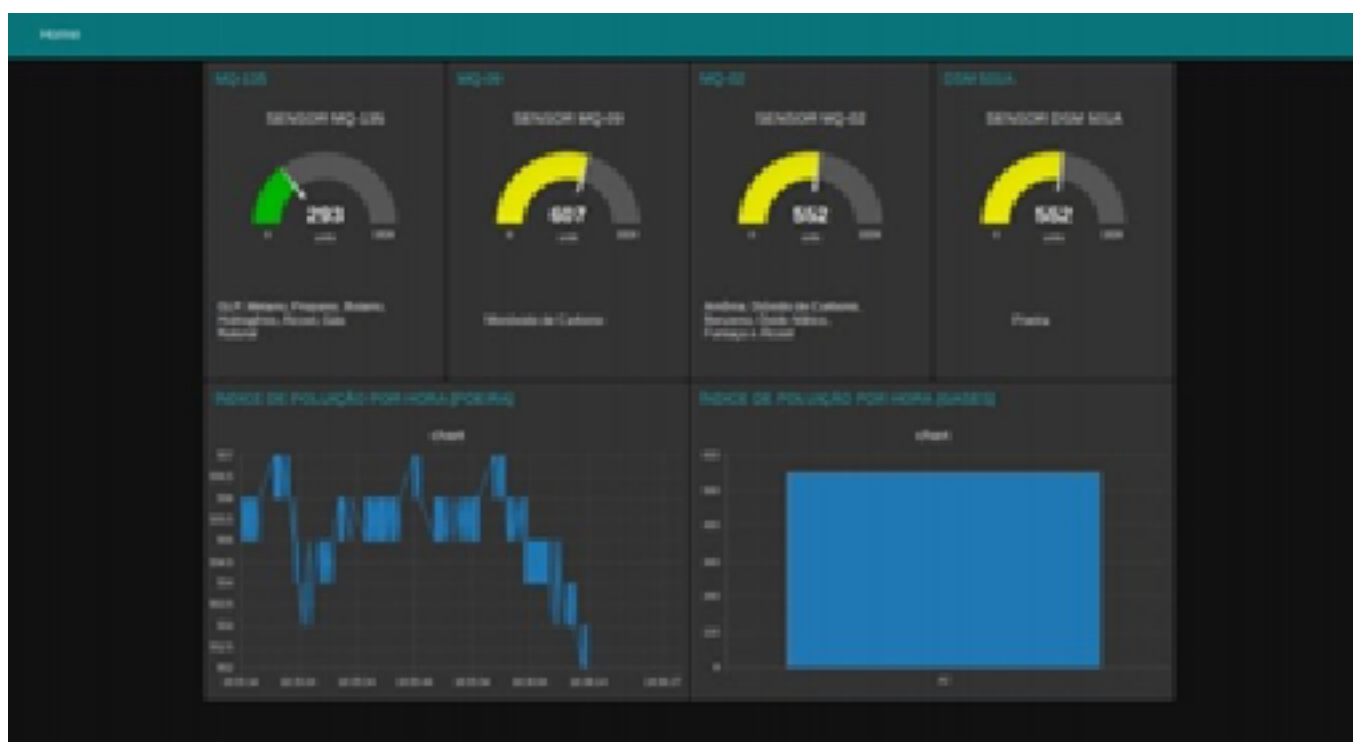

Figura 4. Dashboard do sistema em funcionamento.

Falohun, A., Oke, A., Abolaji, B., and Oladejo, O. (2016). Dangerous gas detection using an integrated circuit and mq-9. International Journal of Computer Applications, $975,8887$.

Harrington, W. (2015). Learning Raspbian. Packt Publishing Ltd.

Karni, J. and Karni, E. (1995). Gypsum in construction: origin and properties. Materials and Structures, 28(2), 92-100.

Knörig, A., Wettach, R., and Cohen, J. (2009). Fritzing: a tool for advancing electronic prototyping for designers. In Proceedings of the 3rd International Conference on Tangible and Embedded Interaction, 351-358. ACM.

Oakes, D., Douglas, R., Knight, K., Wusteman, M., and McDonald, J. (1982). Respiratory effects of prolonged exposure to gypsum dust. In Inhaled Particles V, 833840. Elsevier.

Rabelo, C.S. (2014). O papel da contabilidade gerencial no desenvolvimento das indústrias extrativistas de gesso em grajaú-ma.

Saad, S.M., Saad, A.R.M., Kamarudin, A.M.Y., Zakaria, A., and Shakaff, A.Y.M. (2013). Indoor air quality monitoring system using wireless sensor network (wsn) with web interface. In 2013 International Conference on Electrical, Electronics and System Engineering (ICEESE), 60-64. IEEE.

Simić, M., Stojanović, G.M., Manjakkal, L., and Zaraska, K. (2016). Multi-sensor system for remote environmental (air and water) quality monitoring. In 2016 24th Telecommunications Forum (TELFOR), 1-4. IEEE.

Sulistiyo, A. and Suryono, S. (2016). Wireless sensor system untuk monitoring konsentrasi debu menggunakan algoritma rule based. Youngster Physics Journal, 5(2), 43-50.

Upton, E. and Halfacree, G. (2014). Raspberry Pi user guide. John Wiley \& Sons.

Zhang, G. and Li, G. (2016). Effects of mineral admixtures and additional gypsum on the expansion performance of sulphoaluminate expansive agent at simulation of mass concrete environment. Construction and Building Materials, 113, 970-978. 